\title{
Socio-demographic and clinical characteristics of benzodiazepine long-term users: Results from a tertiary care center
}

\author{
F. Cosci ${ }^{\text {a,* }}$, G. Mansueto ${ }^{a}$, M. Faccini ${ }^{\text {b }}$, R. Casari ${ }^{\text {b }}$, F. Lugoboni $^{\text {b }}$ \\ a Department of Health Sciences, University of Florence, via di San Salvi 12, 50135, Florence, Italy \\ ${ }^{\mathrm{b}}$ Addiction Unit, Verona University Hospital, piazzale Aristide Stefani 1, 37126, Verona, Italy
}

\begin{abstract}
Objective: The use of benzodiazepines (BDZs) represents a critical issue since a long-term treatment may lead to dependence. This study aimed at evaluating socio-demographic and clinical characteristics of BZD long-term users who followed a detoxification program at a tertiary care center.

Method: Two hundred-five inpatients were evaluated. Socio-demographic (e.g., gender, age, education) and clinical information (e.g., BZD used, dose, reason of prescription) was collected. BZDs dose was standardized as diazepam dose equivalents and was compared via the Defined Daily Dose (DDD). Chi-square, Fisher test, ANOVA and Bonferroni analyses were performed.

Results: Females were more frequently BDZ long-term users than males. Hypnotic BZDs were frequently prescribed for problems different from sleep disturbances. Lorazepam, alprazolam, and lormetazepam were the most prescribed drugs. Lorazepam was more frequently used by males, consumed for a long period, in pills, and prescribed for anxiety. Lormetazepam was more frequently consumed by females with a high school education, having a psychiatric disorder, taken in drops and prescribed for insomnia. Lormetazepam had the highest DDD.

Conclusion: A specific profile of BZD long-term user seems to exist and presents different socio-demographic and clinical characteristics according to the benzodiazepine taken into account.

(C) 2016 Elsevier Inc. All rights reserved.
\end{abstract}

\section{Introduction}

For decades, benzodiazepines (BZDs) have been recommended as the standard treatment of anxiety and insomnia [1-4]. In late 1990s, the most frequent recommendation for the treatment of anxiety disorders changed to a selective serotonin reuptake inhibitor (SSRI) or to a serotonin norepinephrine reuptake inhibitor (SNRI) [5] and BDZs were replaced by newer antidepressants mainly due to the claimed BZD withdrawal, rebound, overuse, and abuse [6]. However, the new SSRI and SNRI classification of withdrawal syndromes, which includes persistent postwithdrawal disorders [7], suggested that newer antidepressants have similar problems of withdrawal and rebound as BZDs. In addition, the literature has provided evidence for a reappraisal of BZDs as first-line pharmacological treatment of anxiety disorders without depression; and major differ-

\footnotetext{
Declaration of interest: No conflict of interests.

* Corresponding author. Tel.: +39 0552755066; fax: +39 0556236047.

E-mail address: fiammetta.cosci@unifi.it (F. Cosci).
}

ences in terms of rebound syndrome, potency, risk of abuse, and pharmacokinetics have been shown among BZDs [6].

Analyses of possible predictors for BZD long-term use show that gender is of minor importance [8,9] although more women use than men and the long-term use is much more common among women than men $[9,10]$. Older age was found to be an important predictor [8-11] as well as previous use [8,9] and low level of education [12]. Patients who obtained BDZ prescriptions from doctors in hospital care and patients who obtained prescriptions from doctors working in different settings continued to use BZDs to a greater extent than those who received prescriptions from private practitioners or health centers doctors alone [9]. Also a combined use of hypnotics together with anxiolytics seems to be a significant factor in frequent or daily use [9]. Alcohol consumption, anxiety and tension have been positively related to long-term use, while exercise seems negatively related to it [13]. In addition, according to a recent survey, BZD hypnotics have been associated with increased risk of high-dose use among long-term users, compared to diazepam; BZD anxiolytics were associated with significantly 
lower risk of high-dose use, the lowest risk being with clobazam and clonazepam; finally, triazolobenzodiazepines (i.e., alprazolam and triazolam) were at risk for high-dose use among continuous users [11]. Thus, not all benzodiazepines seemed to be the same [14].

In this framework, we assessed socio-demographic and clinical characteristics of BZD long-term users who referred to a tertiary care center for detoxification with the aim to verify whether routine data are consistent with the results provided by population surveys.

\section{Methods}

\subsection{Sample and procedure}

BZD dependent patients who consecutively referred to the Addiction Unit (AU) of the Verona University Hospital (Verona, Italy) between January 2003 and December 2014 were evaluated. Exclusion criteria were: lifetime and current substance use disorders, other than nicotine and benzodiazepines; addiction to more than one BZD (cases with more than one BZD long-term use were excluded to prevent interpretive bias and allow a clear identification of a socio-demographic and clinical profile of the long-term users for each benzodiazepine).

Inclusion criteria were: age older than 18 years; diagnosis of BZD dependence according to the DSM-IV criteria [15]; BZD addiction lasting from at least 180 days [16]. Eligible patients provided written informed consent and were evaluated by the medical doctors of the AU via standardized questions already used in the past $[17,18]$ which allowed to collect the following information: demographic characteristics; reasons for BDZ prescription; average daily BZD dose consumed in the last 180 days; duration of BZD use; route of administration; co-occurrent psychiatric disorder(s). The study protocol fully adhered to guidelines of the Ethic Committee of the Verona University Hospital, Verona, Italy.

\subsection{Statistical analyses}

The different BZD dose was compared via the Defined Daily Dose (i.e., 1 DDD is the therapeutic daily dose established by WHO for any drug) [19] and BZDs dose was standardized as diazepam dose equivalents using one of the most accepted conversion tables where $10 \mathrm{mg}$ of diazepam is equal to: $1 \mathrm{mg}$ of lorazepam, $2 \mathrm{mg}$ of lormetazepam, $0.25 \mathrm{mg}$ of triazolam, $3 \mathrm{mg}$ of delorazepam, $0.5 \mathrm{mg}$ of alprazolam [20], $6 \mathrm{mg}$ bromazepam [11], 1.5 etizolam [21,22], $20 \mathrm{mg}$ temazepam [11,23].

Comparisons were performed using the chi-square test and Fisher's exact test for dichotomous variables, and the analysis of variance (ANOVA) plus Bonferroni test as a post-hoc analysis for continuous variables.

Significance levels were set at $\mathrm{p} \leq 0.05$ (two-tailed). All of the analyses were performed using SPSS, version 21.0 [24].

\section{Results}

Two hundred-five subjects were enrolled. Among them 132 were females $(64.4 \%)$ and 73 males $(35.6 \%)$, with a mean age of $46.39 \pm 11.33$ years. Most subjects had a high school diploma $(\mathrm{n}=76,37.1 \%)$, followed by middle school diploma $(\mathrm{n}=55,26.8 \%)$, university degree $(\mathrm{n}=37,18 \%)$, and primary school education $(\mathrm{n}=11,5.4 \%)$. Distribution of marital status included: 81 (50.93\%) married, 76 (47.2\%) unmarried, and $3(1.86 \%)$ widowed. About $54 \%(\mathrm{n}=110$, $55.7 \%)$ of the sample was employed, $16.1 \%(\mathrm{n}=33)$ was unemployed, $20.5 \%(\mathrm{n}=42)$ was retired or housewife. Almost the whole sample $(93.2 \%, \mathrm{n}=191)$ had at least one psychiatric disorder. The most frequent mental illnesses were anxiety or depressive disorders $(\mathrm{n}=151,79.47 \%)$, followed by bipolar disorder $(\mathrm{n}=17,8.94 \%)$, personality disorders $(\mathrm{n}=12,6.31 \%)$, others $(\mathrm{n}=8,4.21 \%)$. The use of BZD lasted $80.87 \pm 79.62$ months.

Among anxiolytic BZDs, lorazepam had the highest rate of use in the total sample, followed by alprazolam. Among hypnotic BZDs, lormetazepam had the highest rate of use in the total sample (Table 1).

Lorazepam and delorazepam were more likely to be consumed by males while lormetazepam was more likely to be consumed by females (Table 1). No difference was found in terms of age (Table 2).

When the distribution by working activity, civil status, and education for each benzodiazepine was evaluated, statistically significant results were found only for education (diazepam: 2 subjects completed the primary school, 1 completed the middle school, and 1 the high school, $\mathrm{p}=$ 0.01 , df $=4$; lormetazepam: 6 subjects completed the primary school, 29 the middle school, 57 the high school, 29 the college, for 3 subjects the information was not available, $\mathrm{p}=0.02, \mathrm{df}=4$ ).

Lorazepam was more likely to be consumed in pills while lormetazepam was more likely to be consumed in drops (Table 1). The $5.36 \%$ of the prescriptions for anxiolytic BZDs was for insomnia and the $24.39 \%$ of prescriptions for hypnotic BZDs was for anxiety (98\% of prescriptions was due to lormetazepam).

Lormetazepam was more frequently prescribed in cases with a diagnosis of at least one psychiatric disorder than those without psychiatric disorders $(\mathrm{n}=13,92.9 \%$ vs $\mathrm{n}=123,64.4 \% ; \mathrm{p}=0.03$ ).

When the benzodiazepines were compared in terms of diazepam equivalent dose and duration of use, no statistically significant results were found. Lorazepam was noted to have the longest duration of use while lormetazepam had the highest DDD (Bonferroni post-hoc $\mathrm{p}<0.001$ ) (Table 2).

\section{Discussion}

The present study shows that BZD long-term users asking for detoxification are females in about two thirds of cases, 


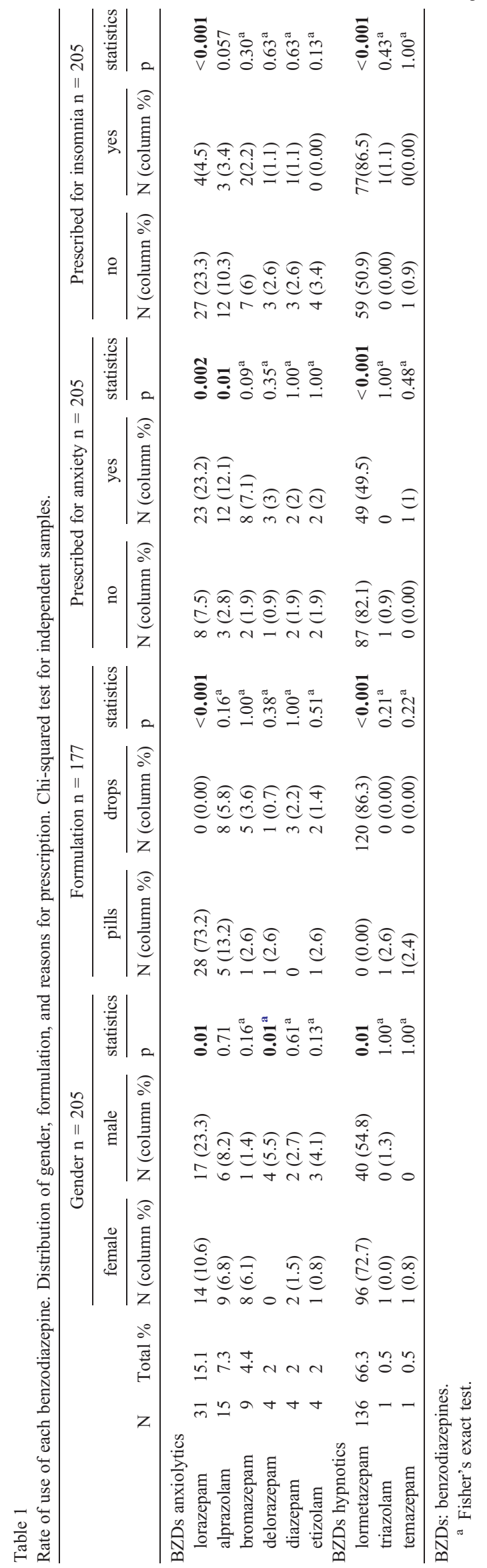

have a high-school level of education in about one third of cases, are married and employed in about half of cases, and have a mean age of about 46 years. The majority of subjects used lorazepam and alprazolam, among anxiolytic BZDs, and lormetazepam, among hypnotic BZDs. The prescription of anxiolytic BZDs was for sleep disturbances rather than for anxiety problems in about $5.36 \%$ of the cases, while the prescription of hypnotics BZDs was for anxiety rather than for sleep disturbances in about $24.39 \%$ of the cases. Among anxiolytic BZDs, lorazepam was more frequently used by males, consumed in pills, and used for a longer period. Even delorazepam was more frequently used by males. Among hypnotic BZDs, lormetazepam was more frequently used by females with a high school education, having a psychiatric disorder, and taken in drops. The highest DDD value was found for lormetazepam.

The findings on gender distribution are consistent with population surveys showing that BZDs intake is twice in women than in men [16,25-29] and with the Luxembourg national registry study which found significantly more high-dose long-term users among women than among men [11]. Interestingly, anxiolytic BZDs were more likely to be used by men while hypnotics BZDs were mostly consumed by women. Unfortunately, no data from the literature evaluated gender distribution among anxiolytic and hypnotic BZDs, thus comparisons on this issue are not possible.

The present sample seems to have a relatively high level of education; this result is only apparently not consistent with the literature [12] since Mant et al. (1988) referred to the general population while we evaluated tertiary care patients, who frequently have an intermediate level of education [30-32].

Among anxiolytic BZDs, lorazepam and alprazolam had the longest-term use while the most hypnotic BZD used was lormetazepam. These findings are consistent with earlier population surveys $[11,18,28,33,34]$ and can be related to the pharmacological characteristics of BZDs which differentiate their dependence potential. Dependence on BZDs following continuous use is most notable for those with a medium elimination half-life such as lorazepam and lormetazepam [35] or for triazolobenzodiazepines, such as alprazolam, which are characterized by high level of lipid solubility [6].

The present research also reported a higher rate of prescriptions, which apparently do not match the indication (i.e., improper prescription), for hypnotics BZDs than for anxiolytic BZDs. Although it is widely known that BDZs may be prescribed improperly, the extent of this problem remains uncertain [36] since studies aimed at evaluating the pattern of prescription of BDZs have yielded mixed results. On one hand, a trend to prescribe anxiolytic BDZs mostly for anxiety disorder and hypnotic BDZs mostly for insomnia was shown [37]; on the other hand, some authors found that both anxiolytics and hypnotics are prescribed for sleep disorders and/or anxiety disorders [38,39]. It is also noteworthy that most of these studies $[27,34,40,41]$ assessed BZDs as a whole rather than estimating the pattern of prescription of each BDZ. 
Table 2

Descriptive (mean $\pm \mathrm{SD}$ ) of continuous variables. Analysis of variance (ANOVA) plus Bonferroni test was applied for age, Defined Daily Dose, diazepam equivalent dose, and duration of treatment.

\begin{tabular}{|c|c|c|c|c|c|c|c|c|c|c|c|c|c|c|}
\hline & \multicolumn{3}{|c|}{$\begin{array}{l}\text { Age (years) } \\
\mathrm{N}=203\end{array}$} & \multicolumn{2}{|c|}{$\begin{array}{l}\text { Dose (mg) } \\
\mathrm{N}=202\end{array}$} & \multicolumn{3}{|c|}{$\begin{array}{l}\text { Times } \times \text { Defined Daily } \\
\text { Dose } N=202\end{array}$} & \multicolumn{3}{|c|}{$\begin{array}{l}\text { Diazepam equivalent dose } \\
\qquad \mathrm{N}=201\end{array}$} & \multicolumn{3}{|c|}{$\begin{array}{l}\text { Duration of use (months) } \\
\qquad \mathrm{N}=198\end{array}$} \\
\hline & mean & SD & $\mathrm{p}$ & mean & SD & mean & $\mathrm{SD}$ & $\mathrm{p}$ & mean & SD & $\mathrm{p}$ & mean & SD & $\mathrm{p}$ \\
\hline \multicolumn{15}{|l|}{ BZDs anxiolytics } \\
\hline lorazepam & 46.87 & 9.48 & & 29.13 & 30.71 & 13.10 & 16.47 & & 291.29 & 307.11 & & 118.97 & 123.49 & \\
\hline alprazolam & 46.93 & 9.68 & & 14.27 & 10.36 & 14.27 & 10.36 & & 285.33 & 207.26 & & 74.93 & 71.26 & \\
\hline bromazepam & 43.89 & 8.58 & & 152.83 & 258.82 & 15.29 & 25.88 & & 305.56 & 517.73 & & 77.11 & 60.58 & \\
\hline delorazepam & 40.50 & 12.71 & & 15.67 & 13.58 & 5.23 & 4.52 & & 53.33 & 45.09 & & 55.50 & 51.23 & \\
\hline diazepam & 40.25 & 17.15 & 0.45 & 85.00 & 75.05 & 8.50 & 7.51 & $<0.001$ & 85.00 & 75.06 & 0.26 & 76.50 & 109.69 & 0.19 \\
\hline etizolam & 37.25 & 10.21 & & 29.50 & 24.17 & - & - & & - & - & & 44.00 & 27.71 & \\
\hline \multicolumn{15}{|l|}{ BZDs hypnotics } \\
\hline lormetazepam & 47.05 & 11.90 & & 65.45 & 67.66 & 65.45 & 67.66 & & 338.35 & 29.12 & & 75.54 & 68.15 & \\
\hline triazolam & 39 & - & & 5 & - & 20 & - & & 100 & - & & 8 & - & \\
\hline temazepam & 48.00 & - & & 13.00 & - & - & - & & 1 & - & & 72.00 & - & \\
\hline
\end{tabular}

BZDs: benzodiazepines.

Both anxiolytic and hypnotic BZDs seemed to be used mainly by patients with intermediate education. This finding can be explained by the fact that the sample under study mainly included subjects having a medium-high level of education.

Finally, lormetazepam had a higher DDD than lorazepam. The result is consistent with the literature [11] and, as already suggested [18], some features may make lormetazepam long-term use easier than that of other BDZs (i.e., high therapeutic index, formulation in drops having a $95 \%$ concentration of alcohol) [18].

Some limitations should be taken into account: (a) psychiatric diagnoses were formulated without a diagnostic tool, due to the use of routine data; (b) even though the sample was large, subgrouping according to the BZD type resulted in relatively small subgroups. Thus, we could infer a pattern of use only for the more largely used BZDs. However, in spite of the small subgroups, the presence of statistically significant results underlines the strength of the results.

On the basis of the present findings, some recommendations could be argued both in research and in clinical practice. From the research point of view, studies evaluating factors identifying subjects at risk to become long-term BZD users and replication studies are warranted. In addition, studies evaluating hypnotic and anxiolytic BZDs separately, and, when possible, each BZD per se, are needed. From a clinical point of view, BZDs should be prescribed on the basis of their pharmacokinetic and pharmacodynamic profiles, not being all the same [14]. In addition, cognitive behavioral therapy [3] should be considered as alternative treatment for those at risk of becoming long-term users. Finally, pros and cons of BZDs recommendation should be balanced under the light of behavioral toxicity and iatrogenic comorbidity [42-48]. Indeed, a drug within its dose range may produce alterations in mood, perceptual, cognitive, and psychomotor functions that can limit the capacity of the individual or constitute a hazard to his/her well-being (i.e., behavioral toxicity) $[42-44,46]$; in addition, a drug treatment can modify the course, characteristics, and responsiveness of the illness for which it was administered (i.e., iatrogenic comorbidity) [45-48].

\section{Acknowledgment}

None.

\section{References}

[1] Rosenbaum JF. The drug treatment of anxiety. Med 1982;306:401-4.

[2] Shader RI, Greenblatt DJ. Use of benzodiazepines in anxiety disorders. Med 1993;328:1398-405.

[3] Smith MT, Perlis ML, Park A, Smith MS, Pennington J, Giles DE, et al. Comparative meta-analysis of pharmacotherapy and behavior therapy for persistent insomnia. Psychiatry 2002;159:5-11.

[4] Offidani E, Guidi J, Tomba E, Fava GA. Efficacy and tolerability of benzodiazepines versus antidepressants in anxiety disorders: a systematic review and meta-analysis. Psychother Psychosom 2013;82:355-62.

[5] Uhlenhuth EH, Balter MB, Ban TA, Yang K. International study of expert judgment on therapeutic use of benzodiazepines and other psychotherapeutic medications: VI. Trends in recommendations for the pharmacotherapy of anxiety disorders, 1992-1997. Depress Anxiety 1999;9:107-16

[6] Chouinard G. Issues in the clinical use of benzodiazepines: potency, withdrawal, and rebound. J Clin Psychiatry 2004;65:7-12.

[7] Chouinard G, Chouinard VA. New classification of selective serotonin reuptake inhibitor withdrawal. Psychother Psychosom 2015;84(2):63-71.

[8] Isacson D, Carsjö K, Bergman U, Blackburn JL. Long-term use of benzodiazepines in a Swedish community: an eight year follow-up. J Clin Epidemiol 1992;45:429-36.

[9] Isacson D. Long-term benzodiazepine use: factors of importance and the development of individual use patterns over time-a 13-year follow-up in a Swedish community. Soc Sci Med 1997;44:1871-80

[10] van Hulten R, Leufkens HG, Bakker A. Usage patterns of benzodiazepines in a Dutch community: a 10-year follow-up. Pharm World Sci 1998;20:78-82.

[11] Cloos JM, Bocquet V, Rolland-Portal I, Koch P, Chouinard G. Hypnotics and triazolobenzodiazepines - best predictors of high-dose 
benzodiazepine use: results from the Luxembourg National Health Insurance Registry. Psychother Psychosom 2015;84:273-83.

[12] Mant A, Duncan-Jones P, Saltman D, Bridges-Webb C, Kehoe L, Lansbury G, et al. Development of long term use of psychotropic drugs by general practice patients. BMJ 1988;296:251-4.

[13] Nordfjærn T, Bjerkeset O, Bratberg G, Moylan S, Berk M, Gråwe R. Socio-demographic, lifestyle and psychological predictors of benzodiazepine and z-hypnotic use patterns. Psychiatry 2014;68:107-16.

[14] Cosci F, Guidi J, Balon R, Fava GA. Clinical methodology matters in epidemiology: not all benzodiazepines are the same. Psychother Psychosom 2015;84:262-4.

[15] American Psychiatric Association. Diagnostic and statistical manual of mental disorders. 4th ed. Washington DC: Author; 2000.

[16] Zandstra SM, Furer JW, de Lisdonk V, van't HM, Bor JH, van Weel C, et al. Different study criteria affect the prevalence of benzodiazepine use. Soc Psychiatry Psychiatr Epidemiol 2002;37:139-44.

[17] Quaglio G, Pattaro C, Gerra G, Mathewson S, Verbanck P, Des Jarlais DC, et al. High dose benzodiazepine dependence: description of 29 patients treated with flumazenil infusion and stabilised with clonazepam. Psychiatry Res 2012;198:457-62.

[18] Faccini M, Leone R, Pajusco B, Quaglio G, Casari R, Albiero A, et al. Lormetazepam addiction: data analysis from an Italian medical unit for addiction. Risk Manag Healthc Policy 2012;5:43-8.

[19] WHO Collaborating Centre for Drug Statistics Methodology (WHOCC). DDD definition and general considerations. Retrieved the 6th of November 2015, http://www.whocc.no/ddd/definition_and_general_considera/.

[20] Gallanter M, Kleber HD, Brady KT. The American Psychiatric Publishing textbook of substance abuse treatment. Arlington: American Psychiatric Pub; 2014.

[21] Inada $T$, Inagaki A. Psychotropic dose equivalence in Japan. Psychiatry Clin Neurosci 2015;69:440-7.

[22] Fukami G, Hashimoto T, Shirayama Y, Hasegawa T, Watanabe H, Fujisaki $\mathrm{M}$, et al. Effects of etizolam and ethyl loflazepate on the P300 event-related potential in healthy subjects. Ann Gen Psychiatry 2010;9:37.

[23] Taylor D, Paton C, Kapur S. The Maudsley prescribing guidelines in psychiatry. Oxford: John Wiley \& Sons; 2015.

[24] SPSS IBM. Statistical package for social science. USA: International Business Machines Corporation SPSS Statistics; 2012.

[25] Holm E, Fosbol E, Pedersen H, Wang TY, Mathews R, Kober L, et al. Benzodiazepine use in Denmark 1997-2008. Eur Geriatr Med 2012;3:299-303.

[26] Magrini N, Vaccheri A, Parma E, D'Alessandro R, Bottoni A, Occhionero $\mathrm{M}$, et al. Use of benzodiazepines in the Italian general population: prevalence, pattern of use and risk factors for use. Clin Pharmacol 1996;50:19-25.

[27] Olfson M, King M, Schoenbaum M. Benzodiazepine use in the United States. JAMA Psychiatry 2015;72:136-42

[28] Vicente Sánchez MP, Macías Saint-Gerons D, de la Fuente HC, González Bermejo D, Montero Corominas D, Catalá-López F. Evolución del uso de medicamentos ansiolíticos e hipnóticos en España durante el período 2000-2011. Rev Esp Salud Publica 2013;87:247-55.

[29] Van Der Waals FW, Mohrs J, Foets M. Sex differences among recipients of benzodiazepines in Dutch general practice. BMJ 1993;307:363-6.

[30] Verma R, Mina S, Deshpande SN. An analysis of paramilitary referrals to psychiatric services at a tertiary care center. Ind Psychiatry J 2013;22:54-9.
[31] Gounder DS, Dhakshinamoorthy A, Nagarajan P, Narasimhan B. Socio-demographic and clinical profile of HIV seropositives in tertiary care teaching hospital of South India. IAIM 2014;4:42-8.

[32] Farhat S, Hussain SS, Rather YH, Hussain SK. Sociodemographic profile and pattern of opioid abuse among patients presenting to a deaddiction centre in tertiary care hospital of Kashmir. J Basic Clin Pharm 2015;6:94-7.

[33] Furtado C, Teixeira I. Utilização de benzodiazepinas em Portugal continental (1999-2003). Acta Med Port 2006;19:239-46.

[34] Petitjean S, Ladewig D, Meier CR, Amrein R, Wiesbeck GA. Benzodiazepine prescribing to the Swiss adult population: results from a national survey of community pharmacies. Int Clin Psychopharmacol 2007;22:292-8.

[35] Kassam A, Patten SB. Hypnotic use in a population-based sample of over thirty-five thousand interviewed Canadians. Popul Health Metr 2006; $4: 15$.

[36] Donoghue J, Lader M. Usage of benzodiazepines: a review. Psychiatry Clin Pract 2010;14:78-87.

[37] Roehrs T, Roth T. Hypnotic'prescription patterns in a large managedcare population. Sleep Med 2004;5:463-6.

[38] Kazim M, Abar A, Hanif M, Mansoor S, Tahir S, Mehmood A, et al. Pattern of benzodiazepine prescription in internal medicine outpatients at a tertiary care hospital in Pakistan. Postgrad Med Inst 2015;29:38-42.

[39] Pélissolo A, Notides C, Lépine JP, Bisserbe JC. Anxiolytic and hypnotic use by general hospital inpatients: the impact of psychopathology and general medical conditions. Gen Hosp Psychiatry 1999;21:79-86.

[40] Donato F, Samani F, Marini MG, Leggieri G, Balestrieri M, Gelatti U. Cross-sectional survey on benzodiazepine use among older people in an Italian region. Public Health 2012;2:42-8.

[41] Ahmer S, Salamat S, Khan RA, Iqbal SP, Haider II, Khan AS, et al. Pattern of benzodiazepine use in psychiatric outpatients in Pakistan: a cross-sectional survey. Clin Pract Epidemiol Ment Health 2009;5:9.

[42] Di Mascio A, Shader RI. Behavioral toxicity of psychotropic drugs: I. Definition. II. Toxic effect on psychomotor function. Conn Med 1968;32:617-20

[43] Di Mascio A, Shader RI. Behavioral toxicity of psychotropic drugs: III. Effect on perceptual and cognitive functions. IV. Effect on emotional (mood) states. Conn Med 1968;32:771-5.

[44] Di Mascio A, Shader RI. Behavioral toxicity of psychotropic drugs: V effects on gross behavioral patterns. Conn Med 1968;33:279-81.

[45] Fava GA, Staccini L, Delle Chiaie R, Belaise C, Tomba E. Farmacopsicologia Clinica. Riv Psichiatr 2014;49:251-4.

[46] Offidani E, Fava GA, Sonino N. Iatrogenic comorbidity in childhood and adolescence: new insights from the use of antidepressant drugs. CNS Drugs 2014;28:769-74.

[47] Fava GA, Gatti A, Belaise C, Guidi J, Offidani E. Withdrawal symptoms after selective serotonin reuptake inhibitor discontinuation: a systematic review. Psychother Psychosom 2015;84:72-81.

[48] Fava GA, Cosci F, Offidani E, Guidi J (in press). Behavioral toxicity revisited: iatrogenic comorbidity in psychiatric evaluation and treatment. J Clin Psychopharmacol. 